JEAN-MARIE LION

Claude ANDRÉ Roche

\title{
Topologie des hypersurfaces pfaffiennes
}

Bulletin de la S. M. F., tome 124, no 1 (1996), p. 35-59

<http://www.numdam.org/item?id=BSMF_1996_124_1_35_0>

C B Bulletin de la S. M. F., 1996, tous droits réservés.

L'accès aux archives de la revue «Bulletin de la S. M. F. » (http: //smf.emath.fr/Publications/Bulletin/Presentation.html) implique l'accord avec les conditions générales d'utilisation (http://www.numdam.org/ conditions). Toute utilisation commerciale ou impression systématique est constitutive d'une infraction pénale. Toute copie ou impression de ce fichier doit contenir la présente mention de copyright.

\section{Numdam}


Bull. Soc. math. France, 124, 1996, p. 35-59.

\title{
TOPOLOGIE DES HYPERSURFACES PFAFFIENNES
}

\author{
PAR \\ Jean-Marie LiON et Claude André ROCHE (*)
}

\begin{abstract}
RÉSUMÉ. - Nous étudions l'accessibilité et la topologie du «bord» des ensembles pfaffiens. Ces ensembles appartiennent à la classe introduite par A.G. Khovanskii qui est constituée des intersections finies de variétés intégrales d'équations différentielles analytiques et d'ensembles semi-analytiques. On démontre que le «bord» d'un ensemble pfaffien est localement connexe par arcs et qu'il est accessible par des "petits chemins» pfaffiens. En dimension trois, on en déduit une stratification de Whitney de tout ensemble pfaffien.
\end{abstract}

ABSTRACT. - We study the topology of the boundary of sets in a new class of subsets of $\mathbb{R}^{n}$. This class of sets, so called pfaffian sets, was introduced by A.G. Khovanskii. It consists of finite intersections of leaves of real analytic foliations with singularities verifying an additionnal hypothesis. For the boundary of these sets, we prove local arc-connectedness and a curve selection lemma. In the 3-dimensional case a Whitney's stratification is obtained for the closure of each pfaffian set.

\section{Introduction et énoncé des résultats}

La structure locale des sous-ensembles semi-analytiques réels est bien connue depuis les travaux de Lojasiewicz [Lo 1 , [Lo $\mathrm{Lo}_{2}$ (voir aussi [D-S], $\left[\mathrm{B}-\mathrm{M}_{1}\right]$ et $\left.\left[\mathrm{B}-\mathrm{M}_{2}\right]\right)$. A.G. Khovanskii $\left[\mathrm{Kh}_{1}\right],\left[\mathrm{Kh}_{2}\right]$ a dégagé la classe des ensembles pfaffiens. Cette classe permet d'étendre la théorie des semianalytiques à certaines solutions d'équations différentielles analytiques. Les ensembles pfaffiens sont des intersections finies de feuilles «nonspiralantes» de feuilletages analytiques singuliers de codimension un et d'ensembles semi-analytiques. Ces ensembles conservent des propriétés de finitude locale de la géométrie analytique réelle. Plusieurs exposés de ces

(*) Texte reçu le 11 avril 1994, révisé le 15 mars 1995.

J.-M. Lion, Université de Bourgogne, URA CNRS 755, BP 138, 21004 Dijon CEDEX, France. Email : lion@satie.u-bourgogne.fr.

C.A. Roche, Université Paul Sabatier, URA CNRS 1408, 118, route de Narbonne, 31062 Toulouse CEDEX, France. Email : roche@cix.cict.fr.

Classification AMS : 32 C05, 32 C 25, 58 A 99, 14 G 30, 32 B 20, 34 C 35. 
idées se trouvent dans $\left[\mathrm{Kh}_{1}\right],\left[\mathrm{Kh}_{2}\right],\left[\mathrm{Ri}_{1}\right],\left[\mathrm{Ri}_{2}\right],\left[\mathrm{Ri}_{3}\right],\left[\mathrm{M}-\mathrm{R}_{1}\right],\left[\mathrm{M}-\mathrm{R}_{2}\right]$, [To], $\left[\mathrm{Li}_{1}\right]$ et $\left[\mathrm{Li}_{2}\right]$.

L'objet de ce travail est de dégager des propriétés plus fines des ensembles pfaffiens. Dans la partie I, on prouve un lemme du petit chemin pfaffien $\left[\mathrm{Li}_{1}\right],\left[\mathrm{Li}_{2}\right],[\mathrm{Mi}]$ (curve selection lemma) : c'est un résultat sur l'accessibilité $d u$ «bord» $\bar{W} \backslash W$ d'un ensemble pfaffien $W$. Dans la partie II, on démontre que ce «bord» possède des propriétés de finitude topologique et qu'il est localement connexe par arcs. On applique les résultats des parties I et II pour montrer que l'adhérence d'un ensemble pfaffien de $\mathbb{R}^{3}$ possède une stratification satisfaisant aux conditions (a) et (b) de Whitney (partie III).

\subsection{Hypersurfaces pfaffiennes de Rolle.}

Soit $M$ un semi-analytique lisse de $\mathbb{R}^{n}$ : c'est un semi-analytique de $\mathbb{R}^{n}$ qui est une sous-variété analytique. Soit

$$
\omega=a_{1} \mathrm{~d} x_{1}+\cdots+a_{n} \mathrm{~d} x_{n}
$$

une 1-forme analytique définie sur un voisinage ouvert $U$ de $\bar{M}$, intégrable au sens de Frobenius $(\omega \wedge \mathrm{d} \omega \equiv 0)$ et transverse à $M$ : en tout point $x$ de $M$, on a $T_{x} M \not \subset \operatorname{ker} \omega(x)$. En particulier, $M$ ne rencontre pas le lieu singulier de $\omega$,

$$
S(\omega)=\{x \in U ; \omega(x)=0\}=\left\{x \in U ; a_{1}(x)=\cdots=a_{n}(x)=0\right\} .
$$

La 1-forme $\omega$ définit un feuilletage analytique de codimension 1 sur $M$ noté $\mathcal{F}(\omega)_{\mid M}$. Une feuille (ou hypersurface pfaffienne) $V$ de $\mathcal{F}(\omega)_{\mid M}$ est une sous-variété immergée injectivement dans $M$, connexe et maximale pour la propriété suivante : si $x$ est dans $V$ alors $T_{x} V=\operatorname{ker} \omega(x) \cap T_{x} M$. En tout point de $M$ passe une unique feuille de $\mathcal{F}(\omega)_{\mid M}$; cette feuille est de codimension un par rapport à $M$ (intégrabilité de $\omega$ ).

En général, une hypersurface pfaffienne est une sous-variété analytique immergée dans $\mathbb{R}^{n}$ qui ne possède pas les propriétés de régularité des sous-ensembles semi-analytiques (un exemple extrême est celui des feuilles du flot irrationnel sur le tore). Mais un examen attentif des méthodes de Khovanskii $\left[\mathrm{Kh}_{1}\right],\left[\mathrm{Kh}_{2}\right]$ permet de choisir une large classe d'hypersurfaces pfaffiennes pour lesquelles on peut prouver des résultats de finitude $\left[\mathrm{M}-\mathrm{R}_{1}\right],\left[\mathrm{M}-\mathrm{R}_{2}\right]$ et $\left[\mathrm{Li}_{2}\right]$. Ces hypersurfaces pfaffiennes sont caractérisées par la propriété suivante dite propriété de Rolle.

DÉfinition 1.- Soient $V, \omega$ et $M$ comme ci-dessus. Le triplet $(V, \omega, M)$ est une hypersurface pfaffienne de Rolle si tout arc $\mathcal{C}^{1} \gamma:[0,1] \rightarrow M$ d'extrémités dans $V$ est tangent au feuilletage $\mathcal{F}(\omega)_{\mid M}$ en au moins un 
point. On dit alors que $V$ est une feuille de Rolle ou une hypersurface pfaffienne de Rolle.

Remarques.

Dans notre étude, il est fondamental que la 1-forme $\omega$ soit définie sur un voisinage ouvert $U$ de l'adhérence du semi-analytique $M$. En particulier, le lieu singulier $S(\omega)$ peut rencontrer $\bar{M} \backslash M$.

Soit $(V, \omega, M)$ une hypersurface pfaffienne de Rolle et soit $M^{\prime} \subset M$ un semi-analytique lisse de $\mathbb{R}^{n}$ tel que $\omega$ soit transverse à $M^{\prime}$. Si $V^{\prime}$ est une composante connexe de $V \cap M^{\prime}$ alors $\left(V^{\prime}, \omega, M^{\prime}\right)$ est une hypersurface pfaffienne de Rolle.

Si $(V, \omega, M)$ est une hypersurface pfaffienne de Rolle, $V$ est une sousvariété analytique fermée de $M$. En revanche, ce n'est pas en général un sous-ensemble sous-analytique de $\mathbb{R}^{n}$. La courbe

$$
\{(x, \exp (-1 / x)) ; x>0\}
$$

est une hypersurface pfaffienne de Rolle du feuilletage du demi-plan $\{x>0\}$ associé à la 1-forme analytique $\omega=y \mathrm{~d} x-x^{2} \mathrm{~d} y$ mais ce n'est pas un sous-analytique.

Une hypersurface pfaffienne n'est pas toujours de Rolle. Mais dès que $M$ est simplement connexe, toute feuille du feuilletage induit par $\omega$ sur $M$ est

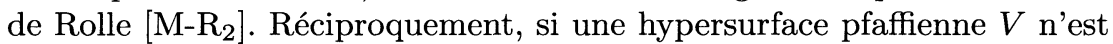
pas de Rolle, il existe un 2-disque analytique immergé dans $M$ sur lequel la trace de $V$ spirale autour d'un polycycle limite du feuilletage induit $\operatorname{par} \omega\left[\mathrm{M}-\mathrm{R}_{2}\right]$.

\subsection{Ensembles pfaffiens et théorème de finitude uniforme.}

Notations. - Fixons le cadre de notre étude. Dans toute la suite, on désigne par :

- $M$ un semi-analytique de $\mathbb{R}^{n}$ lisse et relativement compact;

- $X$ un second semi-analytique $X \subset M$ de $\mathbb{R}^{n}$;

- $\Omega$ une famille $\Omega=\left(\omega_{1}, \ldots, \omega_{q}\right)$ de 1-formes différentielles intégrables, transverses à $M$ et analytiques au voisinage de $\bar{M}$.

DÉfinition 2. - Un ensemble pfaffien $W$ de $M$ décrit à l'aide de $X$ et $\Omega$ est une intersection $W=\left(V_{1} \cap \ldots \cap V_{q}\right) \cap X$ où chaque $\left(V_{i}, \omega_{i}, M\right)$ est une hypersurface pfaffienne de Rolle.

Les résultats de Khovanskii sous la forme adoptée dans [M- $\left.R_{2}\right]$ donnent un sens à cette généralisation. Il découle de $\left[M-R_{2}\right]$ que les ensembles pfaffiens vérifient la propriété de finitude suivante. 
ThÉORÈme DE FINITUde UnIFORMe [M- $\left.\mathrm{R}_{2}\right]$. - Il existe un entier ne dépendant que de $M, X$ et $\Omega$ qui majore le nombre de composantes connexes de tout ensemble pfaffien de $M$ décrit à l'aide de $X$ et de $\Omega$.

\section{3. Énoncé des résultats.}

Le ThÉORÈme I (voir $\left[\mathrm{Li}_{1}\right]$ et $\left[\mathrm{Li}_{2}\right]$ ) joue pour les ensembles pfaffiens le même rôle que celui joué par le lemme du petit chemin en géométrie analytique $\left[\mathrm{B}-\mathrm{M}_{1}\right],\left[\mathrm{B}-\mathrm{M}_{2}\right],[\mathrm{Mi}],\left[\mathrm{Lo}_{1}\right]$ et $\left[\mathrm{Lo}_{2}\right]$.

THÉORÈme I (lemme du petit chemin pfaffien). - Si a est un point non isolé de l'adhérence $\bar{W}$ d'un ensemble pfaffien $W$ de $M$ décrit à l'aide de $X$ et $\Omega$, il existe un arc analytique $\gamma:] 0, \varepsilon[\rightarrow W$ aboutissant en a de façon $\mathcal{C}^{1}$ :

$$
\lim _{t \rightarrow 0^{+}} \gamma(t)=a, \quad \lim _{t \rightarrow 0^{+}} \gamma^{\prime}(t) \in S^{n-1} .
$$

Plus précisément, il existe un semi-analytique lisse $X_{a}$ ne dépendant que de $a, M, X$ et $\Omega$ tel que l'ensemble pfaffien $W \cap X_{a}$ soit une union finie de courbes lisses et $\gamma(] 0, \varepsilon[)$ soit l'une d'elles.

Il sera clair d'après la preuve de ce théorème (voir la partie I) que chaque composante connexe de $W \cap X_{a}$ qui contient le point $a$ dans son adhérence peut être paramétrée analytiquement et aboutit en $a$ avec une tangente.

L'adhérence d'une hypersurface pfaffienne de Rolle $(V, \omega, M)$ au voisinage d'un point non singulier de $\omega$ est un semi-analytique $\left[\mathrm{M}-\mathrm{R}_{2}\right]$. En particulier, si $a$ n'appartient pas à $\bigcup_{k} S\left(\omega_{k}\right)$ le lemme du petit chemin pfaffien (ThÉORÈme I) se réduit au lemme du petit chemin pour les ensembles semi-analytiques. En revanche, au voisinage d'un point singulier de la 1-forme associée, l'adhérence d'une hypersurface pfaffienne de Rolle n'est pas nécessairement sous-analytique. Nous obtenons dans la partie II un résultat qui nous encourage à poursuivre l'étude de la géométrie du «bord» d'une hypersurface pfaffienne de Rolle (voir $\left[\mathrm{Li}_{2}\right]$ et $[\mathrm{C}-\mathrm{L}-\mathrm{M}]$ ). Le voici.

THÉORÈme II. - Il existe un entier ne dépendant que $M, X$ et $\Omega$ qui majore le nombre de composantes connexes du bord $\bar{W} \backslash W$ d'un ensemble pfaffien quelconque $W$ de $M$ décrit à l'aide de $X$ et $\Omega$. De plus ce bord est localement connexe par arcs.

A la suite de ces deux résultats, on peut se demander si le «bord» d'un ensemble pfaffien possède des propriétés de régularité classiques pour les ensembles semi-analytiques. On sait répondre à cette question dans $\mathbb{R}^{3}$. Une première stratification de l'adhérence des hypersurfaces pfaffiennes de Rolle de $\mathbb{R}^{3}$ est donnée dans [M- $\left.\mathrm{R}_{2}\right]$, mais l'obtention d'une stratification

TOME $124-1996-\mathrm{N}^{\circ} 1$ 
vérifiant les conditions de régularité de Whitney nécessite une analyse plus fine : on trouve dans la partie III la preuve du THÉORÈME III.

ThÉORÈme III. - Si $M$ est dans $\mathbb{R}^{3}$ et si $W$ est un ensemble pfaffien de $M$ alors il existe une stratification de Whitney de $\bar{M}$ telle que $W$ est une réunion de strates.

\subsection{Exemples.}

En appliquant le lemme du petit chemin pfaffien (THÉORÈme I) en dimension 2 , on retrouve le résultat suivant. Si $\gamma:]-\infty,+\infty\left[\rightarrow \mathbb{R}^{2}\right.$ est une courbe intégrale d'un champ de vecteurs analytique de $\mathbb{R}^{2}$ et si l'ensemble limite $\bigcap_{t>0} \overline{\gamma(] t,+\infty[)}$ est réduit à un point $a$, alors $\gamma$ spirale autour de ce point ou $\gamma$ admet une tangente en $a$ et la limite

$$
\lim _{t \rightarrow+\infty} \frac{\gamma^{\prime}(t)}{\left\|\gamma^{\prime}(t)\right\|}
$$

existe. Par exemple, la courbe analytique

$$
C=\left\{\left(x, y=\exp \left(-\frac{1}{x}\right)+\exp \left(-\frac{1}{x^{2}}\right) \sin \left(\exp \left(\frac{1}{x^{4}}\right)\right) ; x>0\right\}\right.
$$

n'est pas sous-analytique et le THÉORÈme I entraîne qu'elle n'est tangente à aucun germe de champs de vecteurs analytique bien que son intersection avec tout semi-analytique relativement compact possède un nombre fini de composantes connexes. On aurait pu raisonner aussi par contraposée en appliquant le théorème de finitude uniforme à la forme $y \mathrm{~d} x-x^{2} \mathrm{~d} y$ et à une seconde forme différentielle et en remarquant que le nombre de composantes connexes de $C \cap\{(x, \exp (-1 / x) ; x>0\}$ est infini.

L'exemple suivant donne un aperçu de la complexité de l'adhérence d'une hypersurface pfaffienne de Rolle $(V, \omega, M)$ au voisinage d'un point du lieu singulier $S(\omega)$. Il s'agit d'une hypersurface pfaffienne de Rolle $(V, \omega, M)$ dont le «bord $» \bar{V} \backslash V$ n'est pas un sous-analytique. La 1-forme algébrique

$$
\omega=2 x t z^{2}(y \mathrm{~d} x-x \mathrm{~d} y)+y^{3}\left(t \mathrm{~d} z-z^{2} \mathrm{~d} t\right)
$$

sur $\mathbb{R}^{4}$ admet sur l'ouvert semi-algébrique

$$
M=\{(x, y, z, t) ; y>0, z>0, t>0\}
$$

l'intégrale première $F$ définie par

$$
F(x, y, z, t)=\left(\frac{x}{y}\right)^{2}-\frac{1}{z}-\log t, \quad \text { si } \quad(x, y, z, t) \in M .
$$


Le lieu singulier de $\omega$ est l'ensemble :

$$
S(\omega)=\{y=x z t=0\} \cup\{z=t=0\} .
$$

Le bord de la feuille $F^{-1}(\lambda)$ est l'ensemble :

$$
\overline{F^{-1}(\lambda)} \cap \partial M=\{z=t y=0\} \cup\left\{x=y=0 ; t \geq \mathrm{e}^{-\lambda} \exp (-1 / z)\right\} .
$$

Ce n'est pas un sous-analytique de $\mathbb{R}^{4}$. On remarque que la restriction de $\omega$ à tout 2-plan du type $\left\{z=\mathrm{C}^{\text {te }}, t=\mathrm{C}^{\mathrm{te}^{\prime}}\right\}$ est dicritique (voir [Ce-M] et $[\mathrm{M}-\mathrm{M}])$. On construit d'autres exemples d'hypersurfaces pfaffiennes dont le «bord» est «semi-pfaffien» en considérant l'image réciproque par

$$
G(x, y, z, t)=\left(\left(\frac{x}{y}\right)^{2}-z=u, t\right)
$$

d'une 1-forme

$$
a(u, t) \mathrm{d} u+b(u, t) \mathrm{d} t
$$

qui est polynomiale en $u$ et analytique en $t$.

REMERCIEMENTS. - Les auteurs tiennent à remercier tout particulièrement Robert Moussu qui a suivi ce travail avec beaucoup d'attention. Sans ses nombreux conseils, il n'aurait pas abouti. Nos remerciements vont aussi à Jean-Philippe Rolin. Le premier auteur est chargé de recherche au CNRS.

\section{Lemme du petit chemin Pfaffien}

\section{I.1. Stratification des semi-analytiques et feuillets normaux.}

Nous rappelons ici quelques propriétés locales des ensembles semianalytiques de $\mathbb{R}^{n}$ (voir $\left[\mathrm{Lo}_{1}\right]$ et aussi $[\mathrm{D}-\mathrm{S}]$ ).

Un semi-analytique est un sous-ensemble de $\mathbb{R}^{n}$ tel qu'au voisinage de tout point de $\mathbb{R}^{n}$ il est défini par un nombre fini d'équations et d'inéquations analytiques.

Une stratification analytique $\mathcal{S}$ d'un sous-ensemble $E$ de $\mathbb{R}^{n}$ est une partition localement finie de cet ensemble en sous-variétés analytiques lisses et connexes (appelées strates) vérifiant : si $\Gamma_{1}$ et $\Gamma_{2}$ sont deux strates de $\mathcal{S}$ telles que $\Gamma_{1} \cap \bar{\Gamma}_{2} \neq \emptyset$, alors

$$
\Gamma_{1} \subset \bar{\Gamma}_{2} \text { et } \operatorname{dim} \Gamma_{1}<\operatorname{dim} \Gamma_{2} .
$$

Une stratification :

TOME $124-1996-\mathrm{N}^{\circ} 1$ 
- est dite semi-analytique si elle est analytique et si ses strates sont semi-analytiques;

- est adaptée à un sous-ensemble $F$ si toute strate $\Gamma$ est adaptée à $F$ : $\Gamma \cap F=\emptyset$ ou $\Gamma \subset F$;

- est adaptée à une fonction continue si elle est adaptée à son ensemble de zéros. La fonction est donc de signe constant sur chaque strate.

Une sous-variété analytique de $\mathbb{R}^{n}$ qui est un sous-ensemble semianalytique est appelée semi-analytique lisse.

DÉFINITION 3.-Un feuillet normal $\Gamma$ est un semi-analytique lisse relativement compact de $\mathbb{R}^{n}$ pour lequel il existe des fonctions $f^{+}, f_{m+1}, \ldots, f_{n}$ analytiques au voisinage de $\bar{\Gamma}$ telles que :

1) $\Gamma$ est une composante connexe non vide du semi-analytique

$$
\left\{f^{+}>0\right\} \cap\left\{f_{m+1}=\cdots=f_{n}=0\right\} \cap\left\{\mathrm{d} f_{m+1} \wedge \ldots \wedge \mathrm{d} f_{n} \neq 0\right\},
$$

2) $\bar{\Gamma} \backslash \Gamma \subset\left\{f^{+}=0\right\}$.

Un tel feuillet normal est alors de dimension $m$. Le plan tangent au feuillet normal $\Gamma$ en un point $x \in \Gamma$ est :

$$
T_{x} \Gamma=\bigcap_{i=m+1}^{n} \operatorname{ker} \mathrm{d} f_{i}(x) .
$$

La fonction $f^{+}$mesure la «distance» au «bord» de $\Gamma$. Elle a un rôle semblable à celui des fonctions tapissantes de Thom [Th].

ThÉORÈme DE StRATification Des Semi-AnAlytiques [Lo 1 . - Soit $\left\{E_{i}\right\}_{i \in I}$ une famille localement finie de semi-analytiques de $\mathbb{R}^{n}$. Il existe une stratification semi-analytique de $\mathbb{R}^{n}$ adaptée aux $E_{i}$, pour $i \in I$, telle que les strates soient des feuillets normaux.

Ce théorème est une conséquence de l'étude locale des semi-analytiques que fait Lojasiewicz à l'aide des «partitions normales» [Lo 1 .

\section{I.2. Stratification semi-analytique adaptée à une famille de 1-formes analytiques.}

Les démonstrations et les résultats de ce travail reposent sur la notion de feuillet normal adapté à une famille de 1-formes analytiques. Cette notion traduit la «dégénérescence» de la distribution analytique de plans induite par une famille finie de 1-formes le long d'un feuillet normal. On énonce un lemme d'existence de stratifications en feuillets normaux adaptés à une famille de 1-formes analytiques. 
Soient $M, X$ et $\Omega$ définis précédemment (0.2, notations). Si $\Omega^{\prime} \subset \Omega$ est une sous-famille de $\Omega$, et si $x$ appartient à $\bar{M}$, on note $\operatorname{ker} \Omega^{\prime}(x)$ l'intersection des noyaux $\operatorname{ker} \omega_{j}(x)$ pour $\omega_{j} \in \Omega^{\prime}$ :

$$
\operatorname{ker} \Omega^{\prime}(x)=\bigcap_{\omega_{j} \in \Omega^{\prime}} \operatorname{ker} \omega_{j}(x) .
$$

Définition 4. - Soit $\Gamma$ un semi-analytique lisse inclus dans $\bar{M}$.

- On dit que $\Omega^{\prime} \subset \Omega$ induit le long de $\Gamma$ une ditribution analytique et non-dégénérée de plans $\operatorname{ker} \Omega^{\prime}(x) \cap T_{x} \Gamma$ pour $x \in \Gamma$ si la dimension de $\operatorname{ker} \Omega^{\prime}(x) \cap T_{x} \Gamma$ est constante le long de $\Gamma$.

- On dit que $\Omega^{\prime}$ est transverse $\grave{a} \Gamma$ si en tout point $x$ de $\Gamma$ on a l'égalité

$$
\operatorname{dim}\left(\operatorname{ker} \Omega^{\prime}(x) \cap T_{x} \Gamma\right)=\operatorname{dim} \Gamma-\operatorname{Card} \Omega^{\prime}
$$

où Card $\Omega^{\prime}$ est le nombre d'éléments de $\Omega^{\prime}$.

- Si $\Omega^{\prime}$ est transverse à $\Gamma$ et si pour tout $\Omega^{\prime \prime}$ contenant $\Omega^{\prime}$ et pour tout $x$ de $\Gamma$ on a

$$
\operatorname{ker} \Omega^{\prime \prime}(x) \cap T_{x} \Gamma=\operatorname{ker} \Omega^{\prime}(x) \cap T_{x} \Gamma,
$$

on dit que $\Omega^{\prime}$ est une base de $\Omega$ le long de $\Gamma$.

- La strate $\Gamma$ est adaptée à $\Omega$ si $\Gamma$ est adaptée aux lieux singuliers des $\omega_{i}$ et s'il existe une base $\Omega^{\prime}$ de $\Omega$ le long de $\Gamma$. Les restrictions à $\Gamma$ des familles $\Omega$ et $\Omega^{\prime}$ induisent alors sur $\Gamma$ la même distribution analytique non dégénérée de $k$-plans tangents à $\Gamma$ où $k=\operatorname{dim} \Gamma-\operatorname{Card} \Omega^{\prime}$.

- On dit qu'une stratification de $\bar{M}$ est adaptée à $\Omega$ si ses strates sont adaptées à toute sous-famille de $\Omega$.

Le lemme suivant montre l'intérêt des stratifications semi-analytiques adaptées à $\Omega$.

Lemme I.1. - Soient $\Gamma \subset M$ un semi-analytique lisse adapté à $\Omega$ et $\Omega^{\prime}$ une base de $\Omega$ le long de $\Gamma$. Si $\left(V_{1}, \omega_{1}, M\right), \ldots,\left(V_{q}, \omega_{q}, M\right)$ sont des hypersurfaces pfaffiennes de Rolle, l'ensemble pfaffien

$$
W=\left(V_{1} \cap \ldots \cap V_{q}\right) \cap \Gamma
$$

est une sous-variété analytique fermée de $\Gamma$. Ses composantes connexes sont des sous-variétés analytiques fermées de $\Gamma$ et ce sont des composantes connexes de la sous-variété analytique

$$
\left(\bigcap_{\omega_{j} \in \Omega^{\prime}} V_{j}\right) \cap \Gamma .
$$

De plus ce sont des variétés intégrales de la distribution analytique nondégénérée de plans induite par $\Omega$ (ou par $\Omega^{\prime}$ ) sur $\Gamma$.

La preuve de ce lemme est immédiate.

TOME $124-1996-\mathrm{N}^{\circ} 1$ 
Lemme I.2 (stratification adaptée à des 1-formes). - Soit $\left\{E_{i}\right\}_{i \in I}$ une famille finie de semi-analytiques. Il existe alors une stratification semianalytique de $\bar{M}$ en feuillets normaux adaptée à $M$, aux $E_{i}$ pour $i \in I$, et à la famille $\Omega$.

Ce lemme est un corollaire immédiat de la Proposition 1 de $\left[M-R_{1}\right]$.

Remarque. - On peut supposer que la stratification obtenue dans le Lemme I.2 vérifie les conditions (a) et (b) de Whitney (voir III). En effet, dans la preuve (voir $\left[\mathrm{M}-\mathrm{R}_{1}\right]$ ) on procède par raffinements successifs. A chaque étape, on utilise le théorème de stratification des semianalytiques appliqué à une famille localement finie de semi-analytiques construits à l'aide des 1-formes analytiques $\omega_{j}$. Pour les mêmes raisons on peut supposer que les strates obtenues sont simplement connexes $\left[\mathrm{Lo}_{2}\right]$. Elles ne sont plus alors nécessairement des feuillets normaux.

\section{I.3. Existence d'un petit chemin pfaffien.}

On va démontrer le ThÉORÈme I en deux lemmes. Le premier (LEMME I.3) établit son existence, le deuxième (Lemme I.4) établit que toute courbe pfaffienne aboutit avec une tangente. Ce deuxième résultat se trouve au paragraphe suivant.

Pour $M, X$ et $\Omega$ définis précédemment (0.2, notations), on montre :

Lemme I.3. - Soit a un point de $\bar{M}$. Il existe un semi-analytique $X_{a}$ contenu dans $X$ de dimension au plus $q+1$ tel que si $W$ est un ensemble pfaffien de $M$ décrit à l'aide de $X$ et $\Omega$ et si a est un point non isolé de l'adhérence de $W$, alors l'ensemble pfaffien $W \cap X_{a}$ est une réunion finie de courbes lisses et le point a est un point non isolé de l'adhérence de cette réunion.

Remarquons que $X_{a}$ ne dépend que de $a, X, M$ et $\Omega$ et non pas du choix de $W$.

Preuve. - On construit $X_{a}$ par récurrence sur la dimension de $X$. Si $X$ est de dimension nulle c'est un ensemble fini et la conclusion du lemme est triviale. Supposons maintenant que $X$ soit de dimension $m>0$ et que le Lemme I.3 soit vrai pour tout semi-analytique de dimension strictement inférieure à $m$. D'après le LEMME I.2 (stratification adaptée à des 1-formes), l'ensemble $X$ s'écrit sous la forme

$$
X=Y \cup Z \cup\left(\bigcup_{\mu} \Gamma_{\mu}\right)
$$

où $Y$ est un semi-analytique de dimension strictement inférieure à $m$, $Z$ est un semi-analytique dont l'adhérence ne contient pas $a$ et $\left\{\Gamma_{\mu}\right\}_{\mu \in I}$ 
est une famille finie de feuillets normaux de dimension $m$ adaptés à $\Omega$ et contenant $a$ dans leur adhérence. D'après l'hypothèse de récurrence, il existe un semi-analytique $Y_{a} \subset Y$ de $\mathbb{R}^{n}$ qui répond aux conclusions du LEMme I.3 pour $Y$. Il suffit donc de trouver pour chaque $\Gamma_{\mu}$ un semianalytique $\Gamma_{\mu, a} \subset \Gamma_{\mu}$ qui répond aux conclusions du lemme pour $\Gamma_{\mu}$. Le semi-analytique $X_{a} \subset X$ recherché est la réunion

$$
X_{a}=Y_{a} \cup\left(\bigcup_{\mu} \Gamma_{\mu, a}\right)
$$

Dans la suite on suppose donc que $X$ est un feuillet normal $\Gamma_{\mu}$ de dimension $m$ adhérent à $a$ et adapté à $\Omega$. Soit $\Omega^{\prime}$ une base de $\Omega$ le long de $X$. Quitte à remplacer la famille $\Omega$ par la base $\Omega^{\prime}$, on peut supposer que $\Omega$ est transverse à $X$ et donc $m \geq q$ (théorème de finitude uniforme et LEMme I.2). De plus, on peut supposer que le nombre $q$ de formes de $\Omega$ vérifie $m>q+1$. En effet, si $m=q$, l'ensemble $W$ est fini et l'affirmation est vide. Si $m=q+1, W$ est une réunion finie de courbes par le théorème de finitude uniforme et $X_{a}=X$ convient.

Soient $f_{m+1}, \ldots, f_{n}, f^{+}$les fonctions analytiques définies au voisinage de $\bar{X}$ associées au feuillet normal $X$ (définition 3 ) :

1) $X$ est une composante connexe relativement compacte de l'ensemble

$$
\left\{f^{+}>0\right\} \cap\left\{f_{m+1}=\cdots=f_{n}=0\right\} \cap\left\{\mathrm{d} f_{m+1} \wedge \ldots \wedge \mathrm{d} f_{n} \neq 0\right\}
$$

2) $\bar{X} \backslash X \subset\left\{f^{+}=0\right\}$.

Puisque $\Omega$ est transverse à $X$, la forme analytique au voisinage de $X$,

$$
\eta=\omega_{1} \wedge \ldots \wedge \omega_{q} \wedge \mathrm{d} f_{m+1} \wedge \ldots \wedge \mathrm{d} f_{n}
$$

ne s'annule pas sur $X$ (définition 4). Notons $\delta_{a}$ le carré de la distance euclidienne à $a$.

On déduit alors de 1), de 2), de $\eta_{\mid X} \neq 0$ et de l'inégalité $m>q+1$ l'existence de deux fonctions affines $\ell_{1}$ et $\ell_{2}$, strictement positives sur $\bar{X}$, telles que si on pose $f^{\prime}=\ell_{1} \cdot f^{+}$et $\delta_{a}^{\prime}=\ell_{2} \cdot \delta_{a}$, alors :

$\left.1^{\prime}\right) X \subset\left\{f^{\prime}>0\right\}$

$\left.2^{\prime}\right) \bar{X} \backslash X \subset\left\{f^{\prime}=0\right\}$,

$3^{\prime}$ ) la forme différentielle $\beta=\eta \wedge \mathrm{d} \delta_{a}^{\prime} \wedge \mathrm{d} f^{\prime}$ n'est pas identiquement nulle sur $X$.

TOME $124-1996-\mathrm{N}^{\circ} 1$ 
Ainsi, le lieu des zéros $X_{1}$ de $\beta$ sur $X$ est un semi-analytique de dimension strictement inférieure à celle de $X$. Pour que l'hypothèse de récurrence appliquée a $X_{1}$ permette de prouver le LEMME I.3, il suffit de prouver que si $a$ est dans $\bar{W}$, alors $a$ appartient à $\overline{W \cap X_{1}}$. Supposons donc que $a$ est dans $\bar{W}$. Puisque les composantes connexes de $W$ sont en nombre fini (théorème de finitude uniforme), l'une d'elles au moins contient le point $a$ dans son adhérence. Soit $C$ une composante connexe de $W$ telle que $a \in \bar{W}$. Puisque $X$ est adapté à $\Omega$, la composante connexe $C$ est une sous-variété analytique fermée de $X$ (LEMME I.1). La restriction $\delta_{a \mid C}^{\prime}$ de la fonction $\delta_{a}^{\prime}$ à $C$ est analytique et strictement positive sur la sous-variété analytique connexe $C$. De plus, $a$ appartient à $\bar{C} \backslash C$ et $\delta_{a}^{\prime}(a)=0$. L'image $\delta_{a \mid C}^{\prime}(C)$ est donc un intervalle non vide de la forme $\left.] 0, \lambda_{\infty}\right)$. D'après le théorème de Sard, il existe une suite $\left(\lambda_{\ell}\right)_{\ell \in \mathbb{N}}$ de valeurs régulières de $\delta_{a \mid C}^{\prime}$ dans $\left.] 0, \lambda_{\infty}\right)$ décroissante vers 0 . En prouvant que l'ensemble $C \cap\left\{\delta_{a}^{\prime}=\lambda_{\ell}\right\} \cap X_{1}$ est non vide quelque soit $\ell \in \mathbb{N}$, on aura démontré que $a$ appartient à $\overline{C \cap X_{1}}$ puisque

$$
\bigcap_{\ell \in \mathbb{N}} \overline{\delta_{a}^{\prime-1}\left(\left[0, \lambda_{\ell}\right]\right) \cap X}=\{a\} .
$$

Pour chaque entier naturel $\ell$, le nombre $\lambda_{\ell}$ est une valeur régulière atteinte de $\delta_{a \mid C}^{\prime}$ et l'ensemble $C_{\ell}=C \cap\left\{\delta_{a}^{\prime}=\lambda_{\ell}\right\}$ est une sous-variété analytique fermée de $C$ donc de $X$ ( $C$ est fermé dans $X$ ). Comme $C_{\ell}$ est non-vide et relativement compact, la fonction $f^{\prime}$ est strictement positive, bornée sur $C_{\ell}$ et s'annule sur $\bar{C}_{\ell} \backslash C_{\ell}$. Sa restriction $f^{\prime}{ }_{\mid C_{\ell}}$ atteint son maximum en au moins un point $x_{\ell}$. En ce point on a $\mathrm{d} f^{\prime}{ }_{\mid C_{\ell}}\left(x_{\ell}\right)=0$ et ainsi $x_{\ell}$ appartient à $X_{1}$. Ceci prouve que $a$ est dans $\overline{C \cap X_{1}} \backslash X_{1}$.

\section{I.4. Accessibilité.}

On prouve l'accessibilité du point $a$ en utilisant encore une fois le théorème de finitude uniforme.

Pour $M, X$ et $\Omega$ définis précédemment (0.2, notations) on montre :

Lemme I.4. - Soit $W$ un ensemble pfaffien de $M$ (décrit à l'aide de $X$ et $\Omega$ ) qui est une sous-variété analytique de dimension 1 de $\mathbb{R}^{n}$ et soit a un point de son adhérence $\bar{W}$. Si C est une composante connexe de $W$ telle que $a \in \bar{C}$, il existe une application analytique $\gamma$

$$
\gamma:] 0, \varepsilon[\longrightarrow C
$$

aboutissant en a de façon $\mathcal{C}^{1}$ :

$$
\lim _{t \rightarrow 0^{+}} \gamma(t)=a, \quad \lim _{t \rightarrow 0^{+}} \gamma^{\prime}(t) \in S^{n-1} .
$$

BULLETIN DE LA SOCIÉTÉ MATHÉMATIQUE DE FRANCE 
Preuve. - En reprennant les arguments de la preuve du lemme précédent, on réduit la preuve au cas suivant : $X$ est un feuillet normal de dimension $m, \Omega$ est transverse à $X$ et $m=q+1$.

Il existe des fonctions analytiques au voisinage de l'adhérence du feuillet normal $X, f_{m+1}, \ldots, f_{n}, f^{+}$telles que $X$ soit une composante connexe relativement compacte de l'ensemble

$$
\left\{f^{+}>0\right\} \cap\left\{f_{m+1}=\cdots=f_{n}=0\right\} \cap\left\{\mathrm{d} f_{m+1} \wedge \ldots \wedge \mathrm{d} f_{n} \neq 0\right\} .
$$

La $(n-1)$-forme différentielle $\eta$, analytique au voisinage de $\bar{X}$, définie par

$$
\eta=\omega_{1} \wedge \ldots \wedge \omega_{q} \wedge \mathrm{d} f_{m+1} \wedge \ldots \wedge \mathrm{d} f_{n}
$$

ne s'annule pas sur $X$. L'image de $\eta$ par l'opérateur de dualité euclidienne

$$
\left(\bigwedge_{\substack{j=1 \\ j \neq i}}^{n} \mathrm{~d} x_{j}\right) \longrightarrow(-1)^{i} \frac{\partial}{\partial x_{i}} \quad \text { si } \quad i=1, \ldots, n
$$

est un champ de vecteurs $\vec{U}$, analytique au voisinage de $\bar{X}$, tangent à $X$ et sans singularité sur $X$. Le champ de vecteurs unitaire $\vec{U}_{0}$ défini sur $X$ par

$$
\vec{U}_{0}(x)=\frac{\vec{U}(x)}{\|\vec{U}(x)\|}
$$

est tangent à la sous-variété $W$. Soit $C$ une composante connexe de $W$ dont l'adhérence contient $a$. Le champ $\vec{U}_{0}$ permet de paramétriser la courbe $C$ suivant l'abscisse curviligne. Notons $\gamma:] t_{1}, t_{2}[\rightarrow C$ cette paramétrisation, où $-\infty \leq t_{1}<t_{2} \leq+\infty$. Quitte à restreindre le feuillet $X$ et à changer $\vec{U}$ en $-\vec{U}$ on est ramené à la situation suivante :

$$
a \in \bigcap_{t>t_{1}} \overline{\gamma(] t_{1}, t[)}
$$

Montrons que $\bigcap_{t>t_{1}} \overline{\gamma^{\prime}(] t_{1}, t[)}$ est réduit à un point de $S^{n-1}$.

Cet ensemble est non vide car c'est une intersection décroissante de compacts non vides. Supposons qu'il contienne deux vecteurs distincts $\vec{U}_{1}$ et $\vec{U}_{2}$. Il existe alors deux suites $\left(t_{\ell}\right)_{\ell \in \mathbb{N}}$ et $\left(t_{\ell}^{\prime}\right)_{\ell \in \mathbb{N}}$ décroissantes vers $t_{1}$ et telles que

$$
\lim _{\ell \rightarrow+\infty} \gamma^{\prime}\left(t_{\ell}\right)=\vec{U}_{1} \quad \text { et } \quad \lim _{\ell \rightarrow+\infty} \gamma^{\prime}\left(t_{\ell}^{\prime}\right)=\vec{U}_{2}
$$

Ceci implique que l'intersection du semi-analytique

$$
\left\{x \in X ;\left(\vec{U}_{1}-\vec{U}_{2}\right) \cdot \vec{U}(x)=0\right\}
$$

TOME $124-1996-\mathrm{N}^{\circ} 1$ 
avec la courbe $C$ a une infinité de composantes connexes (on note $\vec{u}_{1} \cdot \vec{u}_{2}$ le produit scalaire des vecteurs $\vec{u}_{1}$ et $\vec{u}_{2}$ ). Ceci est en contradiction avec le théorème de finitude uniforme. Ainsi la limite de $\gamma^{\prime}(t)$ quand $t$ tend vers $t_{1}$ existe. Soit $\vec{u} \in S^{n-1}$ cette limite. L'arc $\gamma$ est de longueur finie, c'est-à-dire $t_{1} \in \mathbb{R}$. Sinon, $t_{1}=-\infty$ et puisque $\gamma^{\prime}(t) \cdot \vec{u}$ a pour limite 1 en $t_{1}$, on aurait :

$$
\lim _{t \rightarrow t_{1}=-\infty} \gamma(t) \cdot \vec{u}=+\infty .
$$

Ceci n'est pas possible car $\gamma(t)$ est dans $X$ qui est relativement compact. On en déduit que $\lim _{t \rightarrow t_{1}} \gamma(t)=a$. Ainsi $\gamma$ arrive au point $a$ de façon $\mathcal{C}^{1}$, tangentiellement au vecteur $\vec{u}$. Ceci achève la preuve de le LEMmE I.4 et donc la preuve du THÉORÈme $\mathrm{I}$.

REMARque. - Le lemme du petit chemin prédédent (ThÉorème I) admet un énoncé plus précis. On appelle cône tangent du sous-ensemble $W$ de $\mathbb{R}^{n}$ au point a l'ensemble suivant :

$$
\begin{array}{r}
\left\{\vec{v} \in S^{n-1} ; \forall \varepsilon>0, \forall \lambda>0, \exists z \in W,\right. \\
\left.\|z-a\|<\varepsilon,\left\|\frac{(z-a)}{\|z-a\|}-\vec{v}\right\|<\lambda\right\} .
\end{array}
$$

Le Lemme I.4 implique que la tangente au point $a$ du «petit chemin pfaffien 》 appartient au cône tangent à $W$ en $a$. Réciproquement, on a (avec les notations de 0.2) :

ThÉORÈme I' (lemme du petit chemin pfaffien). - Soient un point a de $\bar{M}$ et une direction $\vec{u}$ de $S^{n-1}$. Si a est un point non isolé de l'adhérence d'un ensemble pfaffien $W$ de $M$ décrit à l'aide de $X$ et $\Omega$, et si $\vec{u}$ est une direction du cône tangent de $W$ au point $a$, il existe un arc analytique $\gamma:] 0, \varepsilon\left[\rightarrow W\right.$ aboutissant en a de façon $\mathcal{C}^{1}$, tangentiellement $\grave{a} \vec{u}$. De plus, il existe un semi-analytique lisse $X_{a}$ ne dépendant que de $a, \vec{u}, M, X$ et $\Omega$ tel que $W \cap X_{a}$ soit une union finie de courbes lisses et tel que $\gamma(] 0, \varepsilon[)$ soit l'une d'elles.

Pour obtenir la preuve du THÉORÈME I', il suffit de modifier légèrement les arguments de la preuve du Lemme I.3. Quitte à faire un changement linéaire de coordonnées, on peut supposer que la direction privilégiée du cône tangent est celle du vecteur de coordonnées $(0, \ldots, 0,1)$. On considère ensuite une stratification d'un voisinage de $a$ en feuillets normaux adaptés à la famille $\Omega$, ̀̀ $M$, ̀̀ $X$, à l'ensemble $\bigcap_{i=1}^{n-1}\left\{\left|x_{i}\right| \leq\left|x_{n}\right|\right\}$ et au point $a$. Alors, dans la fin de la preuve du Lemme I.3, il suffit de remplacer la fonction $\delta_{a}$ par la fonction

$$
\left(x_{n}^{2}+\left(x_{n-1} / x_{n}\right)^{2}+\cdots+\left(x_{1} / x_{n}\right)^{2}\right)
$$


et la forme $\beta$ par la forme

$$
x_{n}^{3} \cdot \mathrm{d}\left\{\ell_{2} \cdot\left(x_{n}^{2}+\left(x_{n-1} / x_{n}\right)^{2}+\cdots+\left(x_{1} / x_{n}\right)^{2}\right)\right\} \wedge \mathrm{d} f^{\prime} \wedge \eta .
$$

On obtient alors un petit chemin pfaffien aboutissant avec la tangente voulue.

\section{Locale connexité du bord}

\section{II.1. Topologie du bord ${ }^{\mathrm{a}}$.}

La structure différentiable de l'adhérence d'une hypersurface pfaffienne de Rolle n'est pas connue. La difficulté réside dans l'étude de cette hypersurface au voisinage du lieu singulier de la forme différentielle $\omega$ qui la définit. Cependant, dans certains cas, la géométrie est très simple. Par exemple, au voisinage d'un point $a$ de $S(\omega)$ où $\mathrm{d} \omega(a) \neq 0$, on est en présence d'un phénomène de Kupka-Reeb [Ku], [Re], [Ce-M]. Dans un système local de coordonnées analytique $\left(x_{1}, \ldots, x_{n}\right)$ la forme s'écrit :

$$
\omega=A\left(x_{1}, x_{2}\right) \mathrm{d} x_{1}+B\left(x_{1}, x_{2}\right) \mathrm{d} x_{2} .
$$

Ainsi, une hypersurface pfaffienne tangente au noyau de $\omega$ est le produit par $\mathbb{R}^{n-2}$ d'une feuille du feuilletage induit par $\omega$ dans un 2-plan transverse à $S(\omega)$. En revanche, si $\mathrm{d} \omega(a)=0$, il n'y a plus a priori ce phénomène de trivialisation analytique au-dessus d'un 2-plan. Néanmoins, on conjecture que l'adhérence d'une hypersurface pfaffienne de Rolle a une structure stratifiée sous-pfafienne où sous-pfaffien veut dire union de projections propres d'union d'ensembles pfaffiens. Des résultats récents de [Ca-M] sur l'existence de séparatrices pour des feuilletages holomorphes à singularité non dicritique semblent confirmer cette conjecture [Ca-M], [Ca-Ce], [C-L-M].

Dans cette partie, on étudie l'adhérence d'un ensemble pfaffien $W$ de $M$ décrit à l'aide de $X$ et $\Omega(0.2$, notations). L'ensemble pfaffien $W$ est de la forme

$$
W=\bigcap_{i=1}^{q} V_{i} \cap X,
$$

où les $\left(V_{i}, \omega_{i}, M\right)$ sont des hypersurfaces pfaffiennes de Rolle. On entend par bord d'un sous-ensemble $B \subset \mathbb{R}^{n}$ le sous-ensemble :

$$
\partial B=\bar{B} \backslash B \text {. }
$$

L'ensemble $W$ est un fermé de $X$. Son bord $\partial W$ coïncide avec le sousensemble $\bar{W} \cap \partial X$. Le ThÉorÈme II est donc un corollaire du THÉO-

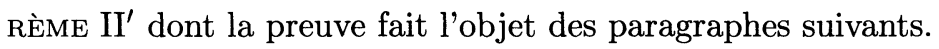

TOME $124-1996-\mathrm{N}^{\circ} 1$ 
THÉORÈme II'. - Si $W$ est un ensemble pfaffien de $M$ décrit à l'aide de $X$ et $\Omega$ et si $Y \subset \bar{M}$ est un semi-analytique de $\mathbb{R}^{n}$, alors $\bar{W} \cap Y$ est localement connexe par arcs et a un nombre fini de composantes connexes. Ce nombre est majoré par un entier ne dépendant que de $M, \Omega, X$ et $Y$.

\section{II.2. Finitude uniforme du nombre de composantes connexes du bord.}

D'après le Lemme I.2, il suffit de prouver le résultat dans le cas où $Y$ est un feuillet normal $\Gamma$ contenu dans $\bar{X}$. Soient $f^{+}, f_{m+1}, \ldots, f_{n}$ les fonctions analytiques associées au feuillet normal $\Gamma$ (définition 3). On pose :

$$
f=f^{+}, \quad g=f_{m+1}^{2}+\cdots+f_{n}^{2} .
$$

L'ensemble $\Gamma$ est une composante connexe de $\{f>0, g=0\}$ et l'on a $\bar{\Gamma} \backslash \Gamma \subset\{f=0\}$. Il existe un ouvert semi-analytique relativement compact $\Delta$ contenant $\Gamma$ et tel que $f$ et $g$ soient définies au voisinage de $\bar{\Delta}$.

Soient $\varepsilon \in] 0,1[$ et $\eta \in] 0,1[$. On va majorer le nombre de composantes connexes de l'ensemble $W_{\varepsilon, \eta}$ défini par

$$
W_{\varepsilon, \eta}=W \cap \Delta \cap X \cap\left\{f \geq \varepsilon, g^{2} \leq \eta\right\} .
$$

Le raisonnement suivant est analogue à celui fait dans $\left[\mathrm{M}-\mathrm{R}_{2}\right]$ pour déduire le théorème de finitude de Gabrielov-Hardt-Teissier du théorème de finitude uniforme. On ajoute deux nouvelles variables $\varepsilon^{\prime}$ et $\eta^{\prime}$ associées aux paramètres $\varepsilon$ et $\eta$ : on considère les feuilletages induits sur

$$
\left.M^{\prime}=M \times\right] 0,1\left[^{2}\right.
$$

par la famille de 1-formes

$$
\Omega^{\prime}=\Omega \cup\left\{\mathrm{d} \varepsilon^{\prime}, \mathrm{d} \eta^{\prime}\right\}
$$

et on considère le semi-analytique

$$
X^{\prime}=\left\{\left(x, \varepsilon^{\prime}, \eta^{\prime}\right) \in(\Delta \cap X) \times\right] 0,1\left[^{2} ; f-\varepsilon^{\prime} \geq 0, \eta^{\prime}-g^{2} \geq 0\right\} .
$$

L'ensemble $W_{\varepsilon, \eta}$ est la projection de l'ensemble pfaffien $W_{\varepsilon, \eta}^{\prime}$ de $M^{\prime}$ décrit à l'aide de $X^{\prime}$ et $\Omega^{\prime}$ :

$$
\left.W_{\varepsilon, \eta}^{\prime}=W \times\right] 0,1\left[^{2} \cap X^{\prime} \cap\left\{\varepsilon^{\prime}=\varepsilon\right\} \cap\left\{\eta^{\prime}=\eta\right\} .\right.
$$

On déduit donc du théorème de finitude uniforme appliqué à $M^{\prime}, X^{\prime}$ et $\Omega^{\prime}$, qu'il existe un entier $N_{\Gamma}$, majorant le nombre de composantes connexes de l'ensemble pfaffien $W_{\varepsilon, \eta}$ indépendamment du choix des réels $\left.\varepsilon, \eta \in\right] 0,1[$ et de l'ensemble pfaffien $W$ de $M$ décrit à l'aide de $X$ et $\Omega$. 
Cette majoration reste vraie si on passe à l'adhérence : le nombre de composantes connexes de $\overline{W_{\varepsilon, \eta}}$ est majoré par $N_{\Gamma}$. Pour $\varepsilon$ fixé, l'ensemble

$$
W_{\varepsilon, 0^{+}}=\bigcap_{\eta>0} \overline{W_{\varepsilon, \eta}}
$$

est une intersection de compacts emboîtés ayant chacun au plus $N_{\Gamma}$ composantes connexes. Par conséquent, le nombre de composantes connexes de $W_{\varepsilon, 0^{+}}$est majoré par $N_{\Gamma}$.

On pose :

$$
K_{\varepsilon, \eta}=\Delta \cap X \cap\left\{f \geq \varepsilon, g^{2} \leq \eta\right\} .
$$

L'ensemble $\Gamma_{\varepsilon}=\Gamma \cap\{f \geq \varepsilon\}$ est une union de composantes connexes de $\bigcap_{\eta>0} \overline{K_{\varepsilon, \eta}}$ et ce dernier contient $W_{\varepsilon, 0^{+}}$. Par conséquent, l'entier $N_{\Gamma}$ majore le nombre de composantes connexes de $\Gamma_{\varepsilon} \cap W_{\varepsilon, 0^{+}}$. Comme l'ensemble $\bar{W} \cap \Gamma$ est l'union de la famille croissante d'ensembles $\Gamma_{\varepsilon} \cap W_{\varepsilon, 0^{+}}$ qui ont chacun au plus $N_{\Gamma}$ composantes connexes, on en déduit que le nombre de composantes connexes de $\bar{W} \cap \Gamma$ est également majoré $\operatorname{par} N_{\Gamma}$.

\section{II.3. Locale connexité par arcs du bord.}

Soit $\Gamma$ un feuillet normal d'une stratification $\mathcal{S}$ de $\bar{X} \cap Y$ en feuillets normaux. Prouvons la locale connexité par arcs de $\bar{W} \cap \Gamma$. Si $a$ appartient à $\Gamma$, il existe $\varepsilon>0$ et $\varepsilon^{\prime}>0$ tels que $\Gamma \cap B\left(a, \varepsilon^{\prime}\right) \subset \Gamma_{\varepsilon}$ où $\Gamma_{\varepsilon}$ est le semi-analytique défini précédemment et $B\left(a, \varepsilon^{\prime}\right)$ la boule euclidienne de centre $a$ et rayon $\varepsilon^{\prime}$. Il suffit donc de prouver le résultat quand $Y$ est le semi-analytique $\Gamma_{\varepsilon}$ avec $\varepsilon>0$. Si $b$ est dans $\Gamma_{\varepsilon}$ et $\varepsilon^{\prime}>0$, l'ensemble

$$
\bar{W} \cap\left(\Gamma_{\varepsilon} \cap B\left(b, \varepsilon^{\prime}\right)\right)
$$

possède un nombre fini de composantes connexes d'après la première partie de la démonstration. L'ensemble $\bar{W} \cap \Gamma_{\varepsilon}$ a localement un nombre fini de composantes connexes. Il est donc localement connexe. Comme il est compact dans un métrique, on conclut à l'aide d'un théorème de Hahn-Moore-Maruskiewicz [Why] qu'il est localement connexe par arcs.

Pour finir, il suffit de prouver qu'au voisinage d'un point $a$ quelconque de $\bar{X} \cap Y$, l'ensemble $\bar{W} \cap Y$ est connexe par arcs. Les feuillets normaux de $\mathcal{S}$ qui contiennent $a$ dans leur adhérence sont en nombre fini. Soit $\Gamma_{\mu}$ un tel feuillet normal. On a déjà prouvé que l'ensemble $\bar{W} \cap \Gamma_{\mu}$ est localement connexe par arcs et possède un nombre fini de composantes connexes. Ces composantes connexes sont donc connexes par arcs. On va conclure en montrant que chaque composante connexe qui contient $a$ dans son adhérence contient un arc continu d'extrémité $a$.

TOME $124-1996-\mathrm{N}^{\circ} 1$ 
Soit $C$ une telle composante connexe. Il existe une suite $\left(C_{k}\right)$ d'ensembles connexes emboîtés contenant le point $a$ dans leur adhérence et tels que $C_{k}$ soit une composante connexe de $C \cap B(a, 1 / k)$. On peut construire cette suite car $a$ appartient à $\bar{C}$ et $C \cap B(a, 1 / k)$ a toujours un nombre fini de composantes connexes. Dans chaque ensemble $C_{k}$, on choisit un point $x_{k}$ : la suite $\left(x_{k}\right)_{k \in \mathbb{N}}$ converge vers $a$. Si $k$ est dans $\mathbb{N}$, l'ensemble $C_{k}$ est connexe par arcs car c'est une composante connexe de

$$
\bar{W} \cap\left(\Gamma_{\mu} \cap B(a, 1 / k)\right)
$$

et $\Gamma_{\mu} \cap B(a, 1 / k)$ est un feuillet normal. Par conséquent, il existe un arc continu

$$
\gamma_{k}:\left[\frac{1}{k+1}, \frac{1}{k}\right] \longrightarrow C_{k}
$$

tel que $\gamma_{k}(1 /(k+1))=x_{k+1}$ et $\gamma_{k}(1 / k)=x_{k}$. Les arcs $\gamma_{k}$ mis bout à bout définissent un arc continu $\gamma:[0,1[\rightarrow C$ en posant $\gamma(0)=a$. Ceci achève la preuve du THÉORÈme II'.

\section{Stratification en dimension trois}

Dans cette dernière partie, on applique les résultats précédents pour prouver l'existence d'une stratification de Whitney de $\mathbb{R}^{3}$ adaptée à une famille localement finie d'hypersurfaces pfaffiennes.

DÉfinition [Wh]. - Une stratification est dite de Whitney si tout couple de strates incidentes $(X, Y)$, avec $X \subset \bar{Y}$, vérifie en tout point de $X$ les conditions suivantes :

- Le couple $(X, Y), X \subset \bar{Y}$, vérifie la condition (a) de Whitney en un point $x \in X$ si

$$
T_{x} X \subset \lim _{n \rightarrow+\infty} T_{y_{n}} Y
$$

pour toute suite $\left(y_{n}\right)_{n \in \mathbb{N}}$ de $Y$ convergeant vers $x$ et telle que la limite de $T_{y_{n}} Y$ existe lorsque $n$ tend vers l'infini.

- Le couple $(X, Y)$, avec $X \subset \bar{Y}$, vérifie la condition (b) de Whitney en un point $x \in X$ si

$$
\lim _{n \rightarrow+\infty} \frac{\overrightarrow{x_{n} y_{n}}}{\left\|\overrightarrow{x_{n} y_{n}}\right\|} \in \lim _{n \rightarrow+\infty} T_{y_{n}} Y
$$

pour toute suite $\left(x_{n}, y_{n}\right)_{n \in \mathbb{N}}$ de $(X, Y)$ convergeant vers $(x, x)$ et telle que les deux limites existent. 
Lojasiewicz démontre que toute stratification semi-analytique de $\mathbb{R}^{n}$ admet un raffinement vérifiant les conditions de Whitney (cf. [ $\left.\left.\mathrm{Lo}_{1}\right]\right)$. Le ThÉORÈme III généralise ce résultat aux sous-ensembles pfaffiens de $\mathbb{R}^{3}$. En effet, on raffine la partition de $\left[\mathrm{M}-\mathrm{R}_{2}\right.$, p. 418] à l'aide de la propriété suivante. On peut «ajouter» une famille localement finie de points $A$ à une stratification $\mathcal{S}$. Il suffit de considérer la collection de ces points augmentée des composantes connexes des $\Gamma \backslash A$ où $\Gamma$ est une strate de $\mathcal{S}$. Si la stratification d'origine vérifiait les conditions de Whitney, il en serait de même de la stratification ainsi augmentée. Enfin, il est à noter que les arguments utilisés dans la preuve du THÉORÈME III ne permettent pas sa généralisation aux dimensions supérieures. On montre le théorème suivant qui implique le ThÉORÈme III de l'introduction :

THÉORÈme III'. - On suppose que $M$ est un semi-analytique lisse relativement compact de $\mathbb{R}^{3}$. Étant donnés $\left(V_{1}, \omega_{1}, M\right), \ldots,\left(V_{q}, \omega_{q}, M\right)$ des hypersurfaces pfaffiennes de Rolle et $X_{1}, \ldots, X_{p}$ des semi-analytiques de $\mathbb{R}^{3}$ inclus dans $\bar{M}$, il existe une stratification de Whitney de $\bar{M}$ adaptée aux hypersurfaces pfaffiennes $V_{1}, \ldots, V_{q}$ et aux semi-analytiques $X_{1}, \ldots, X_{p}$.

La démonstration de ce théorème repose sur les trois lemmes suivants dans lesquels on étudie les conditions de Whitney entre un feuillet normal de dimension 0 ou 1 et une hypersurface pfaffienne de Rolle.

LEMme III.1. - Soit $(V, \omega, M)$ une hypersurface pfaffienne de Rolle de $\mathbb{R}^{3}$ et soit a un point de $\bar{V} \backslash V$. Alors a et $V$ vérifient les conditions de Whitney.

Preuve. - Si $M$ est de dimension 1 ou 2 c'est lemme du petit chemin pfaffien (ThÉorème I). Supposons donc que $M$ est un ouvert semianalytique de $\mathbb{R}^{3}$. Puisque $a$ est un point, $a$ et $V$ vérifient la condition (a). Supposons qu'ils ne vérifient pas la condition (b). Il existe alors un entier $n$ tel que $a$ soit dans l'adhérence de $V \cap X_{n}$ où $X_{n}$ est le semi-analytique

$$
X_{n}=\left\{x \in M ;|\omega(x) \cdot \overrightarrow{x a}| \geq \frac{1}{n}\|\omega(x)\| \cdot\|\overrightarrow{x a}\|\right\}
$$

(si $\eta$ est une $p$-forme, $\|\eta\|^{2}$ est la somme des carrés de ses coefficients). D'après le lemme du petit chemin pfaffien (THÉorème I), il existe un arc $\gamma$ dans $V \cap X_{n}$ aboutissant de façon $\mathcal{C}^{1}$ en $a$. Ceci n'est pas possible : puisque $\gamma(t) \in X_{n} \cap V$, le sinus entre les deux vecteurs $\gamma^{\prime}(t)$ et $\overrightarrow{\gamma(t) a}$ est supérieur à $1 / n$ quelque soit $t$, et ceci contredit la différentiabilité au bout de $\gamma$. Par conséquent, $a$ et $V$ vérifient la condition (b) de Whitney.

Remarque. - Le Lemme III.1 est vrai en toute dimension.

TOME $124-1996-\mathrm{N}^{\circ} 1$ 
Lemme III.2. - Soit $(V, \omega, M)$ une hypersurface pfaffienne de Rolle de $\mathbb{R}^{3}$ et soit $\Gamma_{1}$ un feuillet normal de dimension 1 contenu dans $\bar{V} \backslash V$. L'ensemble des points de $\Gamma_{1}$ où $\Gamma_{1}$ et $V$ ne vérifient pas la condition (a) est fini.

Preuve.-On suppose que $M$ est un ouvert de $\mathbb{R}^{3}$, sinon c'est immédiat. Puisque $\Gamma_{1}$ est un feuillet normal, il existe deux fonctions analytiques $f_{1}$ et $f_{2}$ sur un voisinage $U$ de $\bar{\Gamma}_{1}$ telles que :

$$
\Gamma_{1} \subset\left\{f_{1}=f_{2}=0\right\} \cap\left\{\mathrm{d} f_{1} \wedge \mathrm{d} f_{2} \neq 0\right\} .
$$

L'ensemble $A$ des points de $\Gamma_{1}$ où $\Gamma_{1}$ et $V$ ne vérifient pas (a) est l'union

$$
\bigcup_{n \geq 1}\left(\overline{A_{n} \cap V} \cap \Gamma_{1}\right)
$$

où $A_{n}$ est le semi-analytique suivant :

$$
\begin{aligned}
A_{n}=\left\{x \in U ; \| \mathrm{d} f_{1}(x) \wedge\right. & \mathrm{d} f_{2}(x) \wedge \omega(x) \|^{2} \\
& \left.\geq \frac{1}{n^{2}}\left\|\mathrm{~d} f_{1}(x) \wedge \mathrm{d} f_{2}(x)\right\|^{2} \cdot\|\omega(x)\|^{2}\right\} .
\end{aligned}
$$

Dans $U$, et par dualité euclidienne,

$$
\left\|\mathrm{d} f_{1}(x) \wedge \mathrm{d} f_{2}(x)\right\|^{-1} \mathrm{~d} f_{1}(x) \wedge \mathrm{d} f_{2}(x)
$$

est un vecteur unitaire tangent à des courbes «parallèles》 à $\Gamma_{1}$; de même $\|\omega(x)\|^{-1} \omega(x)$ est un vecteur unitaire normal à la feuille $V$. Donc l'ensemble $A_{n}$ est l'ensemble des points $\xi$ de $U$ où le sinus de l'angle entre une "parallèle » à $\Gamma_{1}$ et le plan $\operatorname{ker} \omega(\xi)$ est supérieur à $1 / n$. Si en un point $\xi_{0}$ de $\Gamma_{1} \cap \bar{V}$ la condition (a) n'est pas vérifiée, le sinus de l'angle entre le vecteur $\mathrm{d} f_{1}\left(\xi_{0}\right) \wedge \mathrm{d} f_{2}\left(\xi_{0}\right)$ et une limite $\lim _{x \rightarrow \xi_{0}} \operatorname{ker} \omega(x)$ n'est pas nul.

Par continuité, il y a un entier $n_{0}$ et des points $\xi$ de $U \cap V$ arbitrairement proches de $\xi_{0}$ tels que $\xi \in A_{n}$ dès que $n>n_{0}$. D'après le THÉORÈme II, il existe un entier majorant uniformément le nombre de composantes connexes de $\overline{A_{n} \cap V} \cap \Gamma_{1}$ pour $n \in \mathbb{N}^{*}$. Pour montrer la finitude de l'ensemble $A$ qui est l'union de la famille croissante d'ensembles $\overline{A_{n} \cap V} \cap \Gamma_{1}$, il suffit donc de montrer que ces ensembles sont finis. D'après le ThÉORÈme II, il suffit de montrer qu'ils ne contiennent pas d'arcs de courbe. On raisonne par l'absurde.

Supposons qu'il existe un entier $n_{1}$ tel que l'ensemble $\overline{A_{n_{1}} \cap V} \cap \Gamma_{1}$ ne soit pas fini. Cet ensemble contient alors un arc $I_{1}$. Quitte à faire un 
changement de coordonnées analytique, à réduire $I_{1}$ et à augmenter $n_{1}$, on se ramène à la situation suivante :

1) $I_{1}$ est l'intervalle

$$
I_{1}=\{(x, y, z) ; 0<x<1, y=z=0\} \quad\left(f_{1}=y, f_{2}=z\right) ;
$$

2) $I_{1}$ est dans l'adhérence de $A_{n_{1}} \cap C \cap D \cap M \cap V$ où $C$ est le coin

$$
\begin{gathered}
C=\{(x, y, z) ; 0<x<1,0<|z|<y<1\}, \\
A_{n_{1}}=\left\{x \in 0 ; a^{2}>\frac{1}{n_{1}^{2}}\left(a^{2}+b^{2}+c^{2}\right)\right\}
\end{gathered}
$$

avec $\omega=a \mathrm{~d} x+b \mathrm{~d} y+c \mathrm{~d} z$ et $D$ le lieu des points où $\omega$ est transverse à la projection orthogonale sur le plan $(x, y)$, c'est-à-dire que $D$ est l'ensemble où la fonction $c$ ne s'annule pas.

Alors, si $\varepsilon>0$, chaque composante connexe $V_{\varepsilon}^{i}$ de

$$
\{y=\varepsilon\} \cap A_{n_{1}} \cap C \cap D \cap V
$$

est le graphe d'une application analytique $g_{\varepsilon}^{i}$ définie sur un intervalle de $I_{1}$ :

$$
\left.g_{\varepsilon}^{i}:\right] a_{\varepsilon}^{i}, b_{\varepsilon}^{i}[\longrightarrow]-\varepsilon, \varepsilon\left[\quad \text { et } \quad V_{\varepsilon}^{i}=\left\{\left(x, \varepsilon, g_{\varepsilon}^{i}(x)\right) ; x \in\right] a_{\varepsilon}^{i}, b_{\varepsilon}^{i}[\} .\right.
$$

L'inégalité $a^{2} \geq \frac{1}{n_{1}^{2}}\left(a^{2}+b^{2}+c^{2}\right)$ pour $x \in A_{n_{1}}$, implique l'inégalité :

$$
\left.\left|\frac{\mathrm{d} g_{\varepsilon}^{i}(x)}{\mathrm{d} x}\right| \geq \frac{1}{n_{1}} \quad \text { pour } \quad x \in\right] a_{\varepsilon}^{i}, b_{\varepsilon}^{i}[.
$$

Puisque $V_{\varepsilon}^{i}$ est contenu dans $C$, cette dernière inégalité implique :

$$
2 \varepsilon \geq\left|g_{\varepsilon}^{i}\left(b_{\varepsilon}^{i}\right)-g_{\varepsilon}^{i}\left(a_{\varepsilon}^{i}\right)\right| \geq \frac{\left|b_{\varepsilon}^{i}-a_{\varepsilon}^{i}\right|}{n_{1}}
$$

Montrons que la double inégalité $\left.{ }^{*}\right)$ contredit le théorème de finitude uniforme : le nombre de composantes connexes des ensembles

$$
\{y=\varepsilon\} \cap A_{n_{1}} \cap C \cap D \cap V
$$

n'est pas majoré par un entier indépendant de $\varepsilon$. Ceci achèvera le raisonnement par l'absurde. Soit $N$ un entier quelconque. D'après $\left(^{*}\right)$, si $\varepsilon$ est inférieur à $1 / 8 n_{1}(N+1)$, chaque intervalle ] $a_{\varepsilon}^{i}, b_{\varepsilon}^{i}$ [ est de longueur strictement inférieure à $1 / 4(N+1)$. D'après le lemme du petit chemin pfaffien (THÉORÈme I), il existe un arc continu $\gamma_{j}$ dans l'ensemble $A_{n_{1}} \cap$ $C \cap D \cap V$ aboutissant au point $a_{j}=(j /(N+1), 0,0)$ si $j=1, \ldots, N$. Si $\varepsilon$ est petit, l'ensemble $\{y=\varepsilon\} \cap A_{n_{1}} \cap C \cap D \cap V$ rencontre tous les arcs $\gamma_{j}$ et des arcs $\gamma_{j}$ distincts rencontrent des composantes connexes différentes de $\{y=\varepsilon\} \cap A_{n_{1}} \cap C \cap D \cap V$. Par conséquent, l'ensemble $\{y=\varepsilon\} \cap A_{n_{1}} \cap C \cap D \cap V$ possède au moins $N$ composantes connexes.

$$
\text { TOME } 124-1996-\mathrm{N}^{\circ} 1
$$


Lemme III.3. - Soit $(V, \omega, M)$ une hypersurface pfaffienne de Rolle de $\mathbb{R}^{3}$ et soit $\Gamma_{1}$ un feuillet normal de dimension 1 contenu dans $\bar{V} \backslash V$ tel qu'en tout point de $\Gamma_{1}, \Gamma_{1}$ et $V$ vérifient la condition (a). Alors l'ensemble des points de $\Gamma_{1}$ où $\Gamma_{1}$ et $V$ ne vérifient pas la condition (b) est fini.

Preuve. - On reprend l'idée de la démonstration du lemme précédent et on suppose encore que $M$ est ouvert. Il existe deux fonctions $f_{1}$ et $f_{2}$, analytiques dans un voisinage $O$ de $\bar{\Gamma}_{1}$ telles que

$$
\Gamma_{1} \subset\left\{f_{1}=f_{2}=0\right\} \cap\left\{\mathrm{d} f_{1} \wedge \mathrm{d} f_{2} \neq 0\right\} .
$$

Quitte à découper $\Gamma_{1}$ et à considérer des coordonnées appropriées, on peut supposer que $\Gamma_{1}$ est le graphe d'une fonction analytique définie de l'intervalle de l'axe des $x:] 0,1[$ dans le carré du plan des $(y, z):] 0,1\left[^{2}\right.$ (en le considérant comme élément d'une partition normale, LEMME I.2). Alors, puisque $\Gamma_{1}$ et $V$ vérifient la condition (a), la recherche des points de $\Gamma_{1}$ où la condition (b) n'est pas vérifiée est équivalente à la recherche des points de $\Gamma_{1}$ où la condition $\left(b^{\pi}\right)$ suivante n'est pas vérifiée $(c f$. $[\operatorname{Tr}])$ :

Condition $\left(b^{\pi}\right)$ POuR $\Gamma_{1}$ ET $V$. L Les sous-variétés $\Gamma_{1}$ et $V$ vérifient la condition $\left(b^{\pi}\right)$ en $a \in \Gamma_{1}$ si pour toute suite de points $\left(y_{n}\right)_{n \in \mathbb{N}}$ de $V$ qui converge vers $a$ :

$$
\lim _{n \rightarrow+\infty} \frac{\overrightarrow{\pi\left(y_{n}\right) y_{n}}}{\left\|\pi\left(y_{n}\right) y_{n}\right\|} \in \lim _{+\infty} T_{y_{n}} V
$$

dès que les limites existent ( $\pi$ est la projection sur $\Gamma_{1}$ parallèlement au plan $(y, z))$.

L'ensemble $B$ des points de $\Gamma_{1}$ où $\Gamma_{1}$ et $V$ ne vérifient pas $\left(b^{\pi}\right)$ est l'ensemble

$$
B=\bigcup_{n \geq 1} \pi_{0}\left(\overline{\widetilde{B}_{n} \cap V \times O} \cap \widetilde{\Gamma}_{1}\right)
$$

où $\pi_{0}: \mathbb{R}^{3} \times \mathbb{R}^{3} \rightarrow \mathbb{R}^{3}$ est la projection canonique et $\widetilde{\Gamma}_{1}$ et $\widetilde{B}_{n}$ sont les semi-analytiques définis par

$$
\begin{aligned}
& \widetilde{\Gamma}_{1}=\left\{\left(m_{1}, m_{1}\right) \in \mathbb{R}^{3} \times \mathbb{R}^{3} ; m_{1} \in \Gamma_{1}\right\} \\
& \widetilde{B}_{n}=\left\{\left(m_{1}, m_{2}\right) \in O^{2} ;\left\|\omega\left(m_{1}\right) \cdot \overrightarrow{m_{1} m_{2}}\right\|^{2} \geq \frac{1}{n^{2}}\left\|\omega\left(m_{1}\right)\right\|^{2} \cdot\left\|\overrightarrow{m_{1} m_{2}}\right\|^{2}\right. \\
& \left.\quad m_{2}=\pi\left(m_{1}\right)\right\} .
\end{aligned}
$$

La définition de ces ensembles reprend les idées de la définition des ensembles $A_{n}$ précédents; ils contiennent les vecteurs de projection qui sont éloignés de l'orthogonal euclidien de la distribution $\operatorname{ker} \omega$. 
D'après le théorème de finitude uniforme, il existe un entier majorant le nombre de composantes connexes de $\overline{\widetilde{B}_{n} \cap V \times O} \cap \widetilde{\Gamma}_{1}$ quel que soit $n$. Pour montrer que $B$ ne possède qu'un nombre fini de points il suffit donc

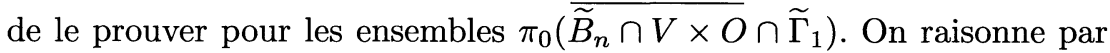
l'absurde.

Supposons qu'il existe un entier $n_{0}$ tel que $\pi_{0}\left(\overline{\widetilde{B}_{n_{0}} \cap V \times O} \cap \widetilde{\Gamma}_{1}\right)$ ne soit pas fini. Il contient alors un arc $I_{0}$. Quitte à faire un changement de coordonnées analytique, à réduire $I_{0}$ et à augmenter $n_{0}$, on se ramène à la situation suivante :

1) $I_{0}$ est l'intervalle

$$
I_{0}=\{(x, y, z) ; 0<x<1, y=z=0\} \quad\left(f_{1}=y, f_{2}=z\right),
$$

2) $I_{0}$ est dans l'adhérence de $B_{n_{0}} \cap V \cap M$ où $B_{n_{0}}$ est le semianalytique

$$
B_{n_{0}}=\left\{m=(x, y, z) \in O ;|b y+c z|^{2} \geq \frac{1}{n_{0}^{2}}\left(a^{2}+b^{2}+c^{2}\right)\left(y^{2}+z^{2}\right)\right\}
$$

avec $\omega=a \mathrm{~d} x+b \mathrm{~d} y+c \mathrm{~d} z$.

Le lemme du petit chemin pfaffien (ThÉorème I) et le LEMme III.1 permettent alors de déduire qu'un point $\left(x_{0}, 0,0\right) \in I_{0}$ ne peut pas être dans l'adhérence de $B_{n_{0}} \cap\left\{x=x_{0}\right\} \cap V \cap M$. Pour $\varepsilon$ petit, le disque

$$
D_{\varepsilon}\left(x_{0}\right)=\left\{(x, y, z) ; x=x_{0} \quad y^{2}+z^{2}<\varepsilon\right\}
$$

ne rencontre pas $B_{n_{0}} \cap V \cap M$. Pour tout entier $N$, il existe $\varepsilon$ tel que les disques $D_{\varepsilon}(j /(N+1))$ ne rencontrent pas $B_{n_{0}} \cap V \cap M$ pour $j$ variant de 1 à $\mathrm{N}$. Puisque $I_{0}$ est dans l'adhérence de $B_{n_{0}} \cap V \cap M \cap\left\{y^{2}+z^{2}<\varepsilon\right\}$, ce dernier a au moins $N$ composantes connexes. Ceci contredit le théorème de finitude uniforme.

Preuve du théorème $I I I^{\prime}$. - On procède en deux étapes. On construit d'abord une stratification en raffinant une stratification semi-analytique de Whitney de $\mathbb{R}^{3}$ en une stratification adaptée aux ensembles semianalytiques et pfaffiens considérés mais qui ne sera plus de Whitney. Ensuite, en appliquant les lemmes précédents, on la raffine en ajoutant un ensemble fini pour vérifier les conditions d'incidence de Whitney.

Soit $\mathcal{S}_{1}$ une stratification semi-analytique de $\bar{M}$ adaptée aux semianalytiques $X_{i}$ et à toute sous-famille de la famille $\Omega=\left(\omega_{1}, \ldots, \omega_{q}\right)$. On choisit $\mathcal{S}_{1}$ vérifiant les conditions de Whitney et avec des strates simplement connexes (voir la remarque après le LEMME I.2). On «ajoutera»

TOME $124-1996-\mathrm{N}^{\circ} 1$ 
à $\mathcal{S}_{1}$ un nombre fini de points. La stratification obtenue sera de Whitney et elle sera adaptée à $\Omega$. On l'appellera encore $\mathcal{S}_{1}$.

Soit $\Gamma_{3}$ une strate de dimension 3 . Si $\left(\omega_{i}, \omega_{j}, \omega_{k}\right)$ est une base de $\Omega$ le long de $\Gamma_{3}$, d'après le théorème de finitude uniforme, l'ensemble $V_{i} \cap V_{j} \cap V_{k} \cap \Gamma_{3}$ est fini. On l'ajoute à $\mathcal{S}_{1}$.

Le bord d'une composante connexe lisse d'un ensemble pfaffien de dimension 1 est composé d'au plus deux points : ses extrémités. Les extrémités des composantes connexes des ensembles $\left(V_{i} \cap V_{j} \cap \Gamma_{3}\right)$, lorsque $\omega_{i}$ et $\omega_{j}$ sont transverses dans $\Gamma_{3}$, forment un ensemble fini de points (ThÉORÈme I et ThÉORÈme II). On les ajoute à $\mathcal{S}_{1}$.

Soit $\Gamma_{2}$ une strate de dimension 2 telle que $\Gamma_{2} \subset \bar{\Gamma}_{3}$. Si $\omega_{i}$ est tangente à $\Gamma_{2}$ alors $\left(\overline{V_{i} \cap \Gamma_{3}}\right) \cap \Gamma_{2}$ est vide. Supposons que $\omega_{i}$ soit transverse à $\Gamma_{2}$. Alors $\left(\overline{V_{i} \cap \Gamma_{3}}\right) \cap \Gamma_{2}$ a un nombre fini de composantes connexes (THÉORÈme II). Soit $C$ l'une d'elles. Alors $\left(C, \omega_{i}, \Gamma_{2}\right)$ est une hypersurface pfaffienne de Rolle $\left(\Gamma_{2}\right.$ est simplement connexe et $\omega_{i}$ est transverse à $\left.\Gamma_{2}\right)$. L'ensemble $\bar{C} \backslash C$ est formée de deux points au plus qu'on ajoute à $\mathcal{S}_{1}$. De plus $C$ vérifie les conditions de Whitney avec chacune de ses extrémités (LEMme III.1). Remarquons enfin que si $C^{\prime}$ est une composante connexe de $V_{i} \cap \Gamma_{3}$ et si $C$ est une composante connexe de $\left(\overline{V_{i} \cap \Gamma_{3}}\right) \cap \Gamma_{2}$ telles que $\overline{C^{\prime}} \cap C$ ne soit pas vide, alors $C$ et $C^{\prime}$ sont incidentes et vérifient les conditions de Whitney (transversalité de $\omega_{i}$ et $\Gamma_{2}$ ).

Soit $\Gamma_{3}^{\prime}$ une seconde strate de dimension 3 telle que $\Gamma_{2} \subset \bar{\Gamma}_{3}^{\prime}$. Si $\left(\omega_{i}, \omega_{j}\right)$ est une base de $\Omega$ le long de $\Gamma_{2}$, alors $\left(\overline{V_{i} \cap \Gamma_{3}}\right) \cap \Gamma_{2}$ et $\left(\overline{V_{j} \cap \Gamma_{3}^{\prime}}\right) \cap \Gamma_{2}$ ont un nombre fini de composantes connexes; chacune d'elles est tangente au feuilletage induit par $\omega_{i}$ sur $\Gamma_{2}$ (pour les premières) ou tangente au feuilletage induit par $\omega_{j}$ sur $\Gamma_{2}$. Elles s'intersectent donc deux à deux transversalement. L'ensemble $\left(\overline{V_{i} \cap \Gamma_{3}}\right) \cap\left(\overline{V_{j} \cap \Gamma_{3}^{\prime}}\right) \cap \Gamma_{2}$ est donc fini. On l'ajoute à $\mathcal{S}_{1}$.

Par ailleurs, si $\Gamma_{1}$ est une strate de dimension 1, les extrémités des composantes connexes de $\Gamma_{1} \cap\left(\overline{V_{i} \cap \Gamma_{3}}\right)$ sont des ensembles finis de points qu'on ajoute à $\mathcal{S}_{1}$.

Après avoir raffiné $\mathcal{S}_{1}$ en rajoutant les points indiqués ci-dessus, on la raffine de la façon suivante. La partition de $\bar{M}$ dont les éléments sont les composantes connexes de

$$
\left(\bigcap_{i \in I} \bar{V}_{i} \cap \Gamma\right) \backslash\left(\bigcup_{j \notin I} \bar{V}_{j}\right), \quad \Gamma \in \mathcal{S}_{1} \quad \text { et } \quad I \subset\{1, \ldots, q\},
$$

est une stratification de $\bar{M}$ qui est adaptée aux hypersurfaces pfaffiennes de Rolle $V_{i}$ et aux semi-analytiques $X_{j}$.

Maintenant, on raffine cette stratification en une stratification de Whitney en lui ajoutant les points, en nombre fini, où $\Gamma_{1}$ et $\left(V_{i} \cap \Gamma_{3}\right)$ ne vérifient 
pas les conditions de Whitney lorsque $\Gamma_{1}$ et $\Gamma_{3}$ sont des strates semianalytiques de dimension 1 et 3 de la stratification précédente (LEMMES III.1, III.2 et III.3). La stratification obtenue est une stratification de Whitney de $\bar{M}$ qui est adaptée aux hypersurfaces pfaffiennes de Rolle $V_{i}$ et aux semi-analytiques $X_{j}$.

\section{BIBLIOGRAPHIE}

[B-M $\mathrm{M}_{1}$ Bierstone (E.) and Milman (P.). - The local geometry of analytic mappings, Universita di Pisa, ETS Editrice Pisa, 1988.

$\left[\mathrm{B}-\mathrm{M}_{2}\right]$ Bierstone (E.) and Milman (P.). - Semianalytic and subanalytic sets, Publi. Math. IHES, t. 67, 1988, p. 5-42.

[Ca-Ce] Cano (F.) and Cerveau (D.). - Desingularization of non dicritical holomorphic foliations and existence of separatrices, Acta Math, t. 169, 1992, p. 1-103.

[C-L-M] Cano (F.), Lion (J.-M.) and Moussu (R.). - Frontière d'une hypersurface pfaffienne, accepté aux Annales de l'École Normale Supérieure en octobre 94.

[Ca-M] Cano (F.) et Mattei (J.-F.). - Hypersurfaces intégrales des feuilletages holomorphes, Ann. Inst. Fourier, t. 42, 1-2, 1992, p. 49-72.

[Ce-M] Cerveau (D.) et Mattei (J.-F.). - Formes intégrables holomorphes singulières, Astérisque, t. 97, 1982.

[D-S] Denkowska (S.) et Stasica (J.). - Ensemble sous-analytiques à la polonaise, preprint, 1985 .

$\left[\mathrm{Kh}_{1}\right]$ Khovanskil (A.G.). - Real analytic varieties with the finitness property and complex abelian integrals, Funct. Anal. and Appl., t. 18, 1984, p. 119-127.

$\left[\mathrm{Kh}_{2}\right]$ KhovanskiI (A.G.). - Fewnomials, AMS Translations of Mathematical Monographs 88, 1991.

[Ku] Kupka (I.). - Singularities of integrable pfaffian forms, Proc. Nat. Acad. Sciences, t. 52, 1964.

[Li $\left.{ }_{1}\right]$ Lion (J.-M.). - Partitions normales de Lojasiewicz et hypersurfaces pfaffiennes, CRAS de Paris, t. 311, série I, 1990, p. 453-456.

[Li $\left.{ }_{2}\right]$ Lion (J.-M.). - Étude des hypersurfaces pfaffiennes, thèse de l'Université de Bourgogne, novembre 1991.

TOME $124-1996-\mathrm{N}^{\circ} 1$ 
[Lo $]$ LojAsiewicz (S.). - Ensembles semi-analytiques, preprint IHES, 1965 .

$\left[\mathrm{Lo}_{2}\right]$ LojAsiewicz (S.). - Triangulation of semi-analytic sets, Ann. Scuola Norm. Sup. Pisa (3), t. 18, 1964, p. 449-474.

[Mi] Milnor (J.). - Singular points of complex hypersurfaces, Ann. of Math. Studies, t. 61, Princeton University Press, 1968.

[M-M] Mattei (J.-F.) et Moussu (R.).- - Holonomie et intégrales premières, Ann. Scien. Éc. Norm. Sup., $4^{e}$ série, t. 13, 1980, p. 469-523.

[M-R 1 Moussu (R.) et Roche (C.A.). - Théorie de Kovanskii et problème de Dulac, Invent. Math., t. 105, 1991, p. 431-441.

$\left[\mathrm{M}-\mathrm{R}_{2}\right]$ Moussu (R.) et Roche (C.A.). - Théorèmes de finitude uniforme pour les variétés pfaffiennes de Rolle, Ann. Inst. Fourier, t. 42, 1-2, 1992, p. 393-420.

[Re] ReEB (G.). - Sur certaines propriétés des variétés feuilletées, Act. Sci. et Ind., Hermann, Paris, $195^{2}$.

[Ri $\left.i_{1}\right]$ Risler (J.-J.). - On the Bézout theorem in the real case, preprint, 1983 .

[Ri $\left.{ }_{2}\right]$ Risler (J.-J.). - Additive complexity and zeros of real polynomials, SIAM J. Comput., t. 14, $\mathrm{n}^{\circ} 1,1985$, p. 178-183.

[Ri $\left.\mathrm{R}_{3}\right]$ Risler (J.-J.). - Complexité et géométrie réelle (d'après A. Khovanskii), Séminaire Bourbaki, t. 637, 1984-85.

[Th] Тном (R.). - Ensembles et morphismes stratifiés, Bull. Amer. Soc. (2), t. 75, 1969, p. 240-284.

[To] Tougeron (J.-C.). - Algèbres analytiques topologiquement nœthériennes, théorie de Kovanskii, Ann. Inst. Fourier, t. 41, 4, 1991, p. 823-840.

[Tr] Trotman (D.). - - Geometric versions of Whitney regularity for smooth stratifications, Ann. Scient. Éc. Norm. Sup., $4^{\mathrm{e}}$ série, t. 12, 1979 , p. $453-463$.

[Wh] Whitney (H.). - Tangents to analytic varieties, Ann. of Math., t. 81, 1965 , p. $496-549$.

[Why] Whyburn (G.T.). - Analytic Topology, AMS Colloquium Pub., t. 28, 1942 . 